Research Article

\title{
Research on Mining of Applied Mathematics Educational Resources Based on Edge Computing and Data Stream Classification
}

\author{
Liping Lu $\mathbb{D}^{1}$ and Jing Zhou ${ }^{2}$ \\ ${ }^{1}$ School of Statistics and Mathematics, Henan Finance University, Zhengzhou 451464, China \\ ${ }^{2}$ Data Analytics Department, Cabin John Consulting Corp., Arlington, VA 22202, USA \\ Correspondence should be addressed to Liping Lu; luliping@hafu.edu.cn
}

Received 27 January 2021; Revised 10 March 2021; Accepted 14 March 2021; Published 26 March 2021

Academic Editor: Hsu-Yang Kung

Copyright (c) 2021 Liping Lu and Jing Zhou. This is an open access article distributed under the Creative Commons Attribution License, which permits unrestricted use, distribution, and reproduction in any medium, provided the original work is properly cited.

Facing the massive data of higher education institutions, data mining technology is an intelligent information processing technology that can effectively discover knowledge from the massive data and can discover important information that people have previously ignored from the huge data information. This article is dedicated to the development of applied mathematics education resource mining technology based on edge computing and data stream classification. First of all, this article establishes a resource system architecture suitable for existing applied mathematics education through edge computing technology, which can effectively improve the efficiency of data mining. Secondly, the data stream classification algorithm is used for information extraction and classification integration of massive applied mathematical education data. This method provides potential and valuable information for decision-makers and education practitioners. Finally, the simulation and performance test of the system verify that it has the functions of mathematical information mining and data processing. This system will provide strong support for applied mathematics education reform.

\section{Introduction}

In recent years, with the rapid development of education, colleges and universities have also developed by leaps and bounds [1, 2]. Colleges and universities are not only expanding the scale of running schools but also increasing the scale of enrollment. With the increase in the number of teachers and the number of students in school, most of the traditional teaching management models have been unable to adapt to the development of schools [3]. In order to effectively improve the efficiency and level of education management, various colleges and universities have increased their investment in informatization, and various information management systems have been continuously used, such as educational administration management system, student achievement management system, student status management system, university personnel management system, and library information management system $[4,5]$. The use of information system greatly improves work efficiency and level [6]. The system saves manpower and material and financial resources and greatly facilitates the daily management of colleges and universities [7]. However, various database systems have accumulated a large amount of data in their use. Over time, these historical data have not been used well. On the contrary, they have become junk files and have been deleted and abandoned $[8,9]$. The reason is that these management systems can realize the functions of data entry, query, and modification, but they cannot discover the internal connections and corresponding rules between these data [10].

With the rapid development of database technology and the wide application of database management systems, how to make full use of data and dig out useful information and knowledge is a question of great concern to organizations with large amounts of data $[11,12]$. Derived from ancient 
data analysis and statistics technology plus modern artificial intelligence, database, and statistics-related technologies, the collection of the above methods realizes interdisciplinary database knowledge discovery, namely, "data mining" $[13,14]$. At present, the application of data mining technology in the field of education management has not yet completed, and universities mainly stay in the use of databases in terms of information construction. Really use data mining technology to mine useful information for education, and obtain very little valuable information [15]. However, with the continuous use of various information management systems in colleges and universities, the amount of data storage is increasing, and the application of data mining technology in the teaching management of colleges and universities must become a trend [16, 17].

If the data from the grassroots can be effectively used, the valuable information hidden in the data can be mined, and the value of the data can be improved, which will provide decision-makers with a better factual basis and basis, thereby changing management methods and formulating better management and formulation to improve the quality and level of running schools $[18,19]$. Facing the massive data of higher education institutions, data mining technology is an intelligent information processing technology that can effectively discover knowledge from the massive data and can discover important information that people have previously ignored from the huge data information. This article is dedicated to the development of applied mathematics education resource mining technology based on edge computing and data stream classification. We discussed the research progress on mathematics education in the second part. The third part discusses the related applications and research of edge computing. The fourth part describes the mining methods of applied mathematics education resources. The fifth part is the actual verification of the case and the performance analysis of the model. In the sixth part, we summarized the full text and looked forward to the future.

\section{Overview of Applied Mathematics Education}

As the main front of mathematics education, ecological research in mathematics classrooms has received more and more attention [20,21]. The previous open teaching of mathematics was mostly limited to the construction of open questions and the development of classroom operations and seldom aimed at the overall research as a model. The development of the application of ecology in the field of education provides us with a new perspective on open teaching $[22,23]$. Therefore, the author combines the research history of education ecology and open teaching of mathematics, analyzes and discusses mathematics classroom teaching from the perspective of ecology, deeply excavates the ecological characteristics of open mathematics teaching, and expounds the feasibility and necessity of mathematics open teaching research. It puts forward a construction strategy and specific implementation process $[24,25]$. The conceptual diagram of applied mathematics education mining is shown in Figure 1.
In educational resources, question banks are very important to educational resources, while traditional question banks focus on providing educators or students with exercises and statistical analysis of students' learning through the right or wrong or scores of the exercises [26, 27]. In order to evaluate the learning situation of students, it cannot conduct effective evaluation and evaluation for individual students nor can it specifically understand the degree of mastery of knowledge points of different students [28, 29]. Therefore, the traditional question bank system cannot provide effective tutoring functions for individual students. In order for the question bank resources to effectively guide students and improve their learning and thinking ability in the process of doing questions, the question bank resources need to contain more knowledge information, so useful knowledge information needs to be dug out from the question bank $[30,31]$. The schematic diagram of the applied mathematics education mining system architecture is shown in Figure 2.

Process ontology is a subtype of ontology. It describes related process models in the domain in a declarative way and needs to express concepts and relationships such as processes, activities, and timing in the domain [32, 33]. Foreign research on process ontology mainly includes the help of OWL description language, proposes a layered network ontology language business-OWL, and applies this language to the decomposition and formulation of dynamic business processes [34]. Celentano et al. proposed the application of process ontology to the exam business process specification framework, which realized the semantic capture and sharing in the $\mathrm{B} 2 \mathrm{~B}$ e-commerce model and was able to perform knowledge deduction and knowledge reasoning on shared information [35]. Professor Fan Yuxin of Tsinghua University and others proposed a design process description metamodel based on extensible markup language XML and process ontology. The model constructs the description rules of the core elements of the process definition language based on extensible markup language to realize the multidisciplinary design optimization process and information. It can be seen that the research of process ontology is closely related to application, but the process ontology constructed in different application fields is also different in representation and structure [36].

\section{Overview of Edge Computing Technology}

The ultralarge bandwidth and extremely low-latency service access of 5G puts new demands on the network [37]. The traditional network structure is a tree structure, and the business layer needs to be converted to a central node for processing, but when facing big data business scenarios, problems arise [38]. When all services are deployed to the central node, the network load is greatly increased and the network delay time is longer, which puts forward higher requirements on network bandwidth and delay performance. In order to solve the problem of limited computing storage space and power consumption of mobile terminals, we believe that it is necessary for $i$ to migrate high-complexity, high-energy-consuming computing tasks to the 


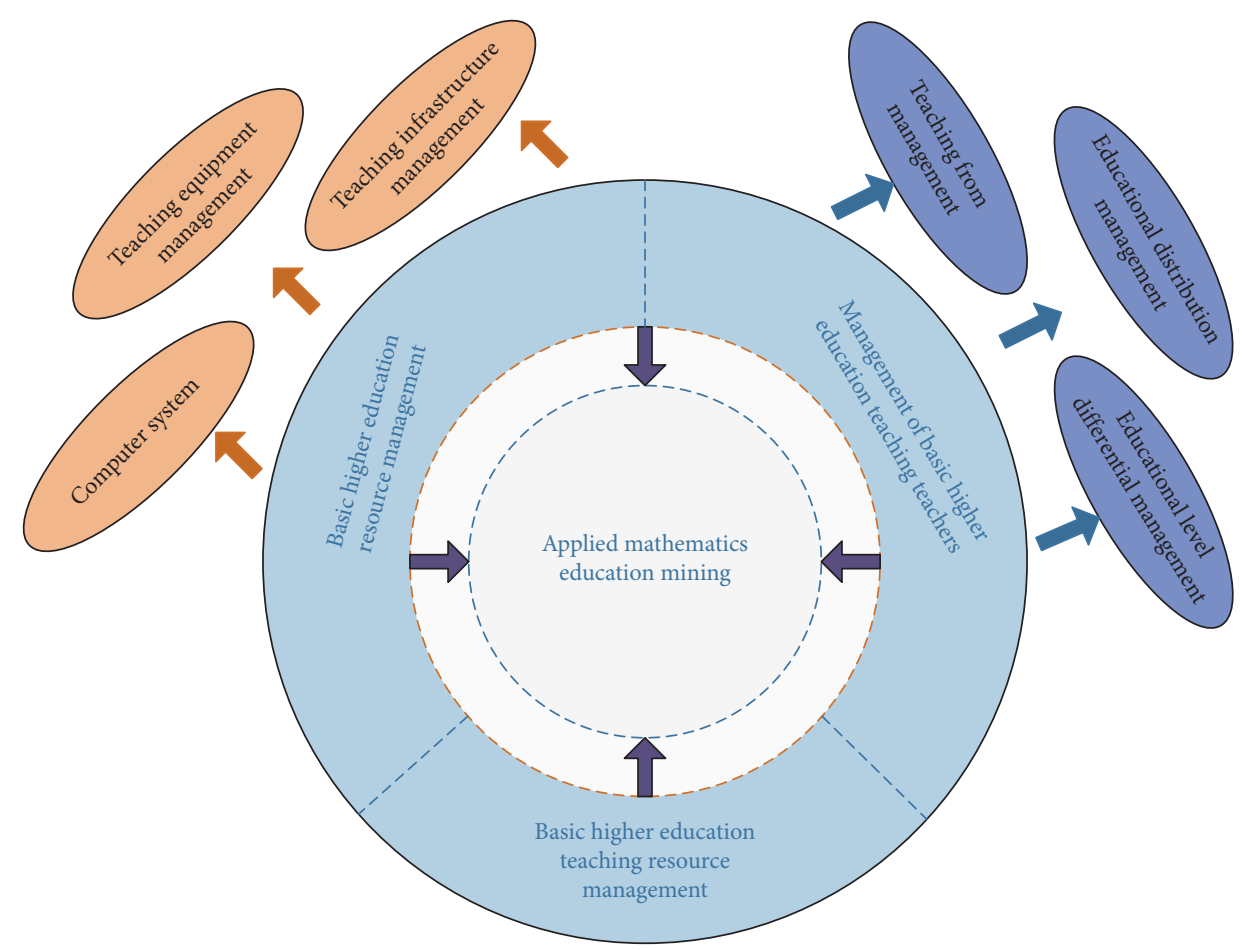

FIGURE 1: Schematic diagram of applied mathematics education mining concept.

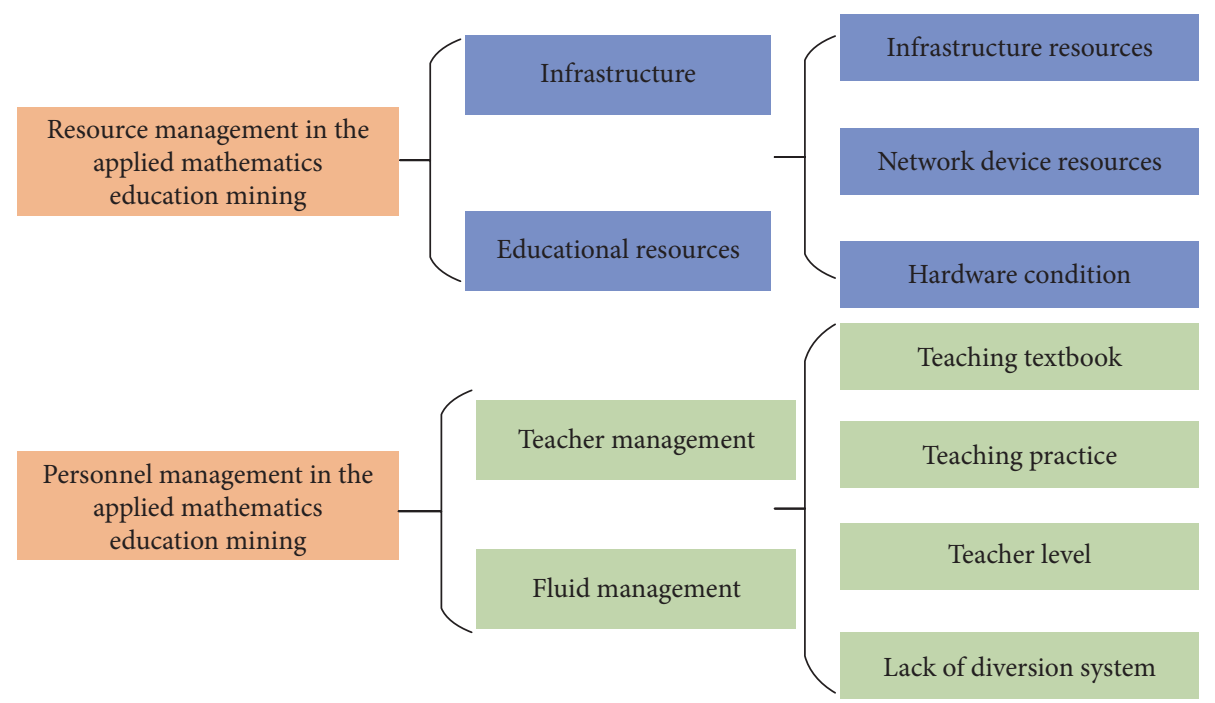

FIGURE 2: Schematic diagram of applied mathematics education mining system architecture.

server side of the cloud computing center to reduce energy consumption. This technology can consume mobile terminals and extend their standby time. However, the method of migrating computing tasks to cloud computing centers also increases the data transmission delay, which has a certain impact on delay-sensitive business applications and QoS. Mobile edge computing provides great possibilities for service innovation at the edge of the network. The conceptual diagram of mobile edge computing is shown in Figure 3.

Mobile edge computing (MEC) can be defined as an implementation of edge computing, which introduces

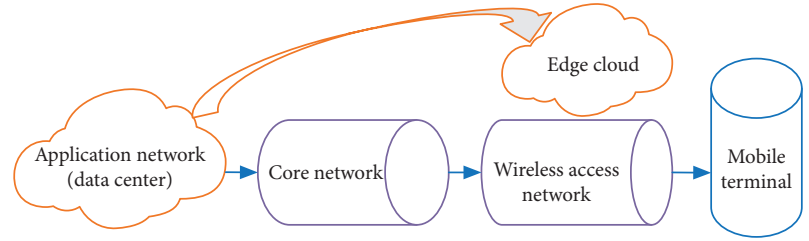

Figure 3: Conceptual diagram of mobile edge computing.

computing and storage functions to the edge of the radio access network, thereby reducing waiting time by moving the cloud and service platform to the edge of the network. As 
shown in Figure 3, using servers deployed at the edge of the network, mobile operators can provide suitable production and operating environments for various upper-layer applications, and "sink" services down to mobile terminals to reduce network congestion. Deploying the application server at the edge of the wireless network can make full use of redundant computing resources at the edge of the network, and mobile devices and IoT devices can achieve low completion delays for computing tasks.

\section{Mining of Applied Mathematics Education Resources}

4.1. Data Stream Classification. Classification technology is the study of how to assign observed samples to predefined categories. In recent years, there have been more and more researches on data stream mining. Compared with static data, data stream data have the characteristics of high speed, unlimited, concept drift, concept evolution, and a small number of tags. According to the type of classifier used, the data stream is classified into a single classification model and an integrated classification model. The data stream contains a lot of information [39].

The concept drift phenomenon in the unstable data stream seriously reduces the classification accuracy, so this paper proposes a dynamic weighting scheme to solve this problem. This scheme explains in detail how to assign weights to all training examples in the data block. The higher the weight of the example, the greater the impact on the classifier training process:

$$
\begin{aligned}
& \operatorname{Pr}[A(X) \in \widehat{D}] \leqq e^{\left[X, X^{\prime}\right]} \operatorname{Pr}\left[A\left(X^{\prime}\right) \in \widehat{D}\right], \\
& \widetilde{p}(n, m)=p(n, m) * h(n)=\sum_{L=-N_{1}}^{N_{1}} p(n-l, m) h(l) .
\end{aligned}
$$

In this way, you can directly control which instances at the current moment should be set with higher weights so that the trained classifier can better adapt to the current concept:

$$
\begin{aligned}
\delta_{i}^{\left(n_{1}\right)} & =\frac{\partial}{\partial z_{i}^{\left(n_{1}\right)}} J(W, b ; x, y), \\
\delta_{i}^{\left(n_{1}\right)} & =\frac{\partial}{\partial z_{i}^{\left(n_{1}\right)}} \frac{1}{2}\left\|y-h_{w, b}(x)\right\|^{2}=-\left(y_{i}-a_{i}^{\left(n_{1}\right)}\right) \cdot f^{\prime}\left(z_{i}^{\left(n_{1}\right)}\right) .
\end{aligned}
$$

The biggest difference between data flow and other data is that data flow is time-sensitive; that is, only newer instances can reflect the trend of data changes. Therefore, the importance of the examples in the data stream for training the classifier will change over time; especially in the case of a nonstationary environment, if a concept drift occurs, the data distribution at the current moment will be significantly different from the previous distribution:

$$
\begin{aligned}
\delta_{i}^{(l)} & =\left(\sum_{j=1}^{S_{l+1}} W_{j i}^{(l)} \delta_{j}^{(l+1)}\right) f^{\prime}\left(z_{i}^{(l)}\right), \\
\widetilde{P}_{2} & =s \cdot P_{2} \cdot R+L .
\end{aligned}
$$

When processing data streams, it is necessary to consider the problem of data stream processing in nonstationary environments. At this time, it is often not feasible to use only naive Bayes classifiers because in nonstationary situations, it is not enough to just deal with the incoming instances. It is also necessary to consider the problem of concept drift and make appropriate responses to it:

$$
\begin{aligned}
v_{i j}^{e+1}= & W+V_{I j}^{e}+C_{1} * R_{1} *\left(\text { Best }_{i j}-P_{i j}^{e}\right) \\
& +C_{2} * R_{2} *\left(g \text { Best }_{i j}-P_{i j}^{e}\right), \\
s= & \operatorname{tr}\left(P_{K 1}^{T} \cdot P_{k 2} \cdot R\right) / \operatorname{tr}\left(P_{K 2}^{T} \cdot P_{k 2}\right) .
\end{aligned}
$$

Since the naive Bayes classifier has no concept drift detection mechanism and the ability to adapt to concept drift, it is not suitable for processing data streams with concept drift. In this case, the classification accuracy of the naive Bayes classifier will be significantly reduced:

$$
\left.\begin{array}{rl}
\tilde{p}(0) & =h(-255) p(-255)+\ldots+h(0) p(0)+\ldots+h(255) p(255), \\
\tilde{p}(0) & =h(-255) p(-255)+\ldots+h(0) p(1)+\ldots+h(255) p(255), \\
\vdots & \\
\tilde{p}(0) & =h(-255) p(-255)+\ldots+h(0) p(255)+\ldots+h(255) p(255),
\end{array}\right\}
$$


With the arrival of data blocks, new tag data may be generated. The appearance of the new mark may be a set of feature values that have not been seen before, or a known mark that has not been seen before, or both. Therefore, both feature and mark space are taken into consideration.

\subsection{Mining of Applied Mathematics Educational Resources} Based on Data Stream Classification. Among educational resources, the question bank is very important to educational resources. The traditional question bank focuses on providing exercises for educators or students, and statistical analysis of students' learning through the scores of the exercises. But it can only be used to evaluate the learning status of students as a whole. It cannot effectively evaluate and evaluate individual students, nor can it specifically understand the degree of mastery of knowledge points of different students.

The displacement of the center of gravity is used to describe the effect of applying mathematical mining under different data stream classification indicators. The classic barycentric coordinate is a coordinate defined on a plane polygon, not limited to a specific coordinate system. The points in the polygon are expressed linearly by the vertices of the polygon, and the combination coefficient is the center of gravity coordinate. In order to generalize the construction method of GBCs, this paper understands the barycentric coordinates from the perspective of linear algebra from the recursion, discusses the restriction conditions when recursing the barycentric coordinates from the two-sided polygon to the $n+1$ polygon, and gives a method for constructing the barycentric coordinates kind of new ideas. It is not limited to those functions that have geometric meaning, as long as a function that satisfies the inequality relationship is constructed, a set of centers of gravity coordinates can be obtained. The displacement of the center of gravity is used as an index to measure the effect of applying mathematics mining under different data stream classification indexes, as shown in Figure 4.

As shown in Figure 4, under different data stream classification indicators, there are great differences in the application of mathematical mining. Therefore, the importance of example data in training the classifier will change over time, especially in nonstationary environments. If a concept drift occurs, the data distribution at the current moment will be different from the previous one. The distribution is significantly different [40].

We have added relevant citations to the revised manuscript. In order to compare the effectiveness and efficiency of the model established in this paper, we compare the model established in this paper with the methods in recent international references [40-42]. Most of the existing data stream adaptive classification models usually assume that the true label can be obtained after the data are input into the classification model to obtain the predicted label. However, this assumption is unreasonable in some cases because data labeling is usually expensive and time-consuming.
Therefore, we use the accuracy of the model as a comparison index to compare the particle swarm and the neural network optimized by ADAM $[43,44]$. The application mathematics mining effect of these three models on the three sample sets is shown in Figure 5.

The experimental data in Figure 5 reflect some of the problems in information mining in applied mathematics education. Compared with static data, data stream data have the characteristics of high speed, unlimited, concept drift, concept evolution and a small number of tags. According to the type of classifier used, the data stream is classified into a single classification model and an integrated classification model. The data stream contains a lot of information. The concept drift phenomenon in the unstable data stream seriously reduces the classification accuracy, so this paper proposes a dynamic weighting scheme to solve this problem. Therefore, the lack of necessary authentication information during the automatic authentication process will cause the automatic authentication to fail. According to data analysis, the most important problem is mainly because the computer cannot effectively understand the information provided by the exercise.

\section{Case Analysis of Mining of Applied Mathematics Educational Resources}

5.1. Sample Data Collection. In educational resources, question banks are very important to educational resources, while traditional question banks focus on providing exercises for educators or students and statistical analysis of students' learning conditions through the correctness and wrongness or scores of the exercises. Through the above research on the edge computing and data stream classification model, the network throughput of the applied mathematics education resource mining system is summarized.

The largest network power consumption is when a large amount of data are sent and received. In order to reduce the power consumption of the terminal device, in actual applications, the data should be sent in a short time as much as possible to avoid frequent waking up of the wireless network card. Based on the above research, we propose a scheme to test the network throughput of the applied mathematics education resource mining system. Figure 6 shows the network test of applied mathematics education system resources in different network environments.

It can be seen from Figure 6 that, under different network environments, the error rate of predicting applied mathematics education system resource network sample datasets is low. It can be seen that the data stream classification technology has effectively improved the quality and classification of original applied mathematics education information data samples. Figure 7 shows the simulation results of applied mathematics education system resource throughput under different test standards.

It can be seen from Figure 7 that, under different test standards, the error rate of predicting the applied 


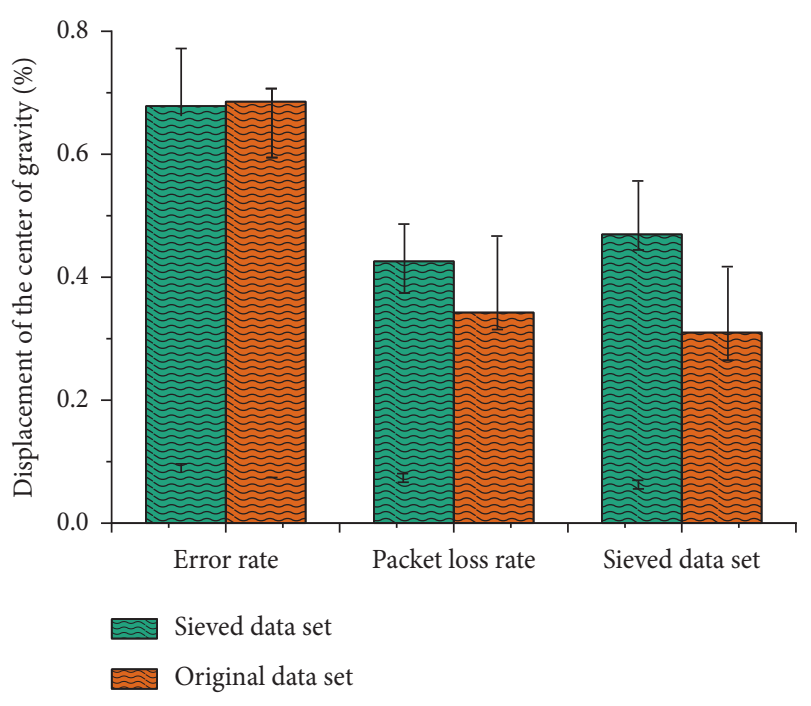

Figure 4: Effect of applied mathematics mining under different data stream classification indicators.

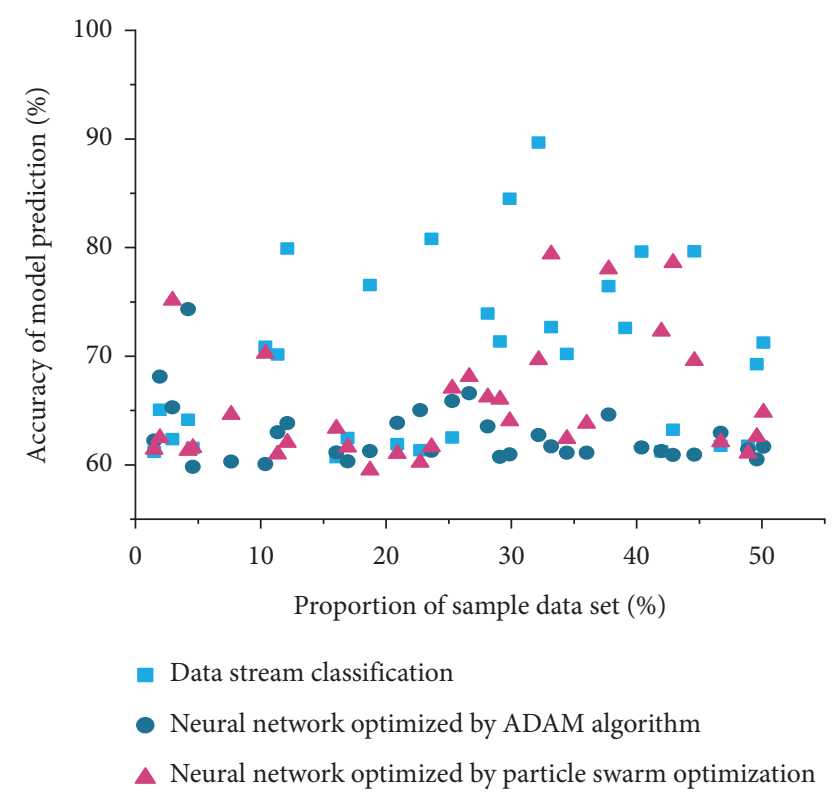

FIGURE 5: Application of mathematics mining effect under different data flow models.

mathematics education system resource network sample dataset is low. It can be seen that the data stream classification technology has effectively improved the quality and classification of original applied mathematics education information data samples. If the data from the grassroots can be effectively used, the valuable information hidden in the data can be mined, and the value of the data can be improved, which will provide decision-makers with a better factual basis and basis, thereby changing management methods and formulating better management and formulation to improve the quality and level of running schools.

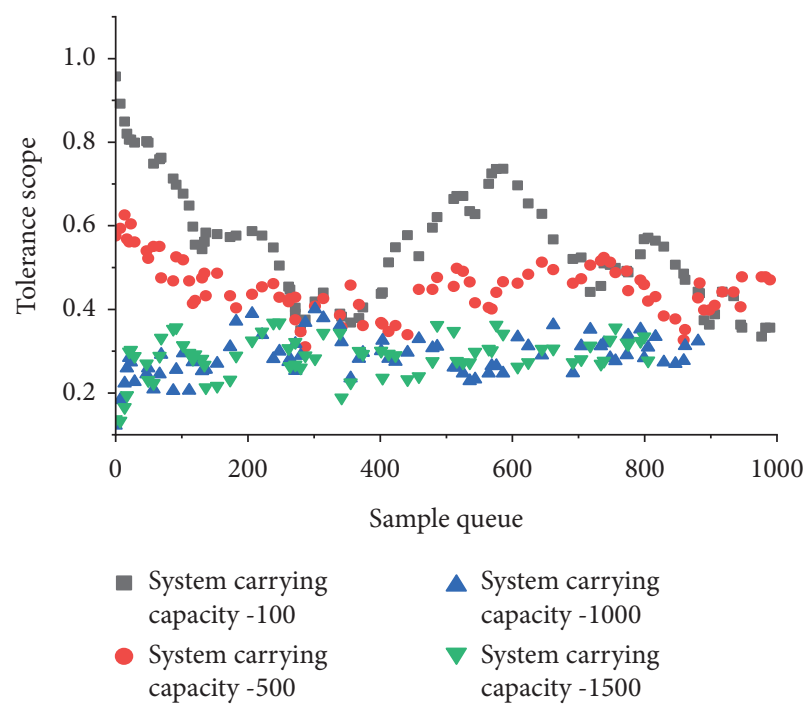

FIGURE 6: Network test of applied mathematics education system resources under different network environments.

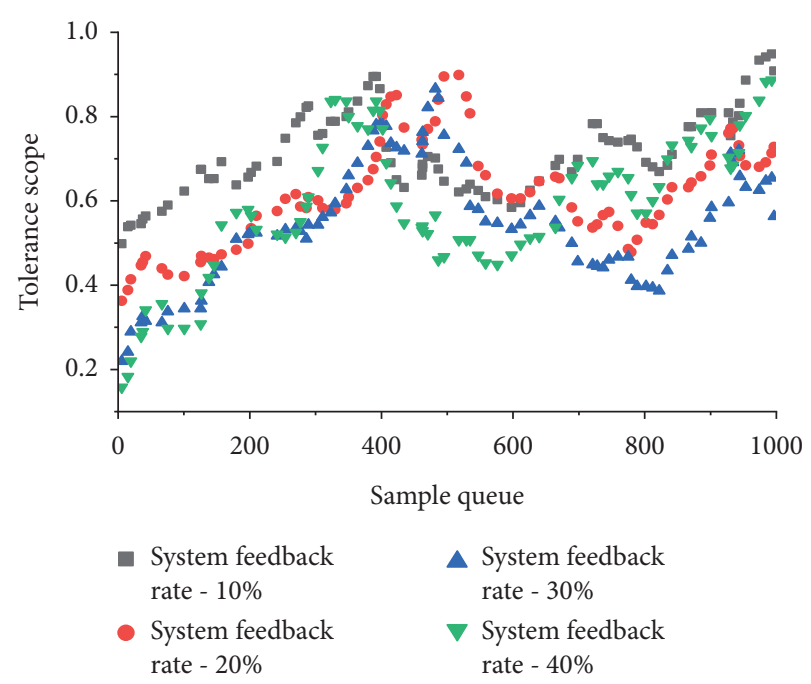

Figure 7: Simulation of resource throughput of applied mathematics education system under different test standards.

\section{Conclusion}

With the rapid development of database technology and the wide application of database management systems, how to make full use of data and dig out useful information and knowledge is a question of great concern to organizations with large amounts of data. At present, the application of data mining technology in the field of education management has not yet completed, and universities mainly stay in the use of databases in terms of information construction. This article is dedicated to the development of applied mathematics education resource mining technology based on edge computing and data stream classification. Due to the 
limitations of our own knowledge, we will be committed to further exploring new mechanisms and methods in related fields to improve the level and quality of existing applied mathematics education resources mining. Facing the massive data of higher education institutions, data mining technology is an intelligent information processing technology that can effectively discover knowledge from the massive data and can discover important information that people have previously ignored from the massive data.

\section{Data Availability}

The datasets used and/or analyzed during the current study are available from the corresponding author on reasonable request.

\section{Disclosure}

This article does not contain any studies with human participants or animals performed by any of the authors. This paper did not receive any funding. All authors agree to submit this version and claim that no part of this manuscript has been published or submitted elsewhere.

\section{Conflicts of Interest}

The authors declare that there are no conflicts of interest.

\section{References}

[1] J. Zhang, L. Zhou, F. Zhou et al., "Computation-efficient offloading and trajectory scheduling for multi-UAV assisted mobile edge computing," IEEE Transactions on Vehicular Technology, vol. 69, no. 2, pp. 2114-2125, 2020.

[2] K. Yue and C. Tayler, "Choosing educational resources to build interprofessional, palliative care competency: a replicable review methodology," Journal of Palliative Care, vol. 13, no. 8, pp. 415-428, 2020.

[3] B. Yang, X. Cao, J. Bassey, X. Li, and L. Qian, "Computation offloading in multi-access edge computing networks: a multitask learning approach," 2020, https://arxiv.org/abs/ 2006.16104.

[4] R. L. Vicente-Vicente, M. Prieto, M. Pescador et al., "The TOX-OER project: learning toxicology through open educational resources. An international experience in permanent education," Toxicology Letters, vol. 280, p. S304, 2017.

[5] N. Tratnik, "Generalized cut method for computing the edgeWiener index," Discrete Applied Mathematics, vol. 282, pp. 222-233, 2020.

[6] G. Tatiana, G. Z. Choleva, D. L. Tsogas, and E.C. Giokas, "An edge-iot framework and prototype based on blockchain and smart contracts," Talanta, vol. 6, pp. 4719-4732, 2019.

[7] A. Tanious, J. D. Brooks, L. J. Wang, M. L. Shames, and D. L. Dawson, "Educational resources for vascular laboratory education in vascular surgery residencies and fellowships: survey of Vascular Surgery Program Directors," Journal of Vascular Surgery, vol. 69, no. 6, 2019.

[8] Z. Sun, C. L. Liu, J. Niu, and W. Zhang, "Discriminative structure learning of sum-product networks for data stream classification," Neural Networks, vol. 123, 2019.

[9] N. Shaikh, R. U. De Azevedo, A. Rajesh, and D. R. Farley, "Re: lack of online video educational resources for open colorectal surgery training," Anz Journal of Surgery, vol. 89, no. 5, p. 618, 2019.

[10] M. Pratama, W. Pedrycz, and G. I. Webb, "An incremental construction of deep neuro fuzzy system for continual learning of nonstationary data streams," IEEE Transactions on Fuzzy Systems, vol. 28, no. 7, pp. 1315-1328, 2020.

[11] L. Peng, H. Zhang, H. Hassan, Y. Chen, and B. Yang, "Accelerating data gravitation-based classification using GPU," The Journal of Supercomputing, vol. 75, no. 6, pp. 2930-2949, 2019.

[12] B. Olav, W. A. Ueland, J. Espen et al., "Acoustic classification in multifrequency echosounder data using deep convolutional neural networks," Ices Journal of Marine Science, vol. 4, p. 4, 2020.

[13] S. Nizamuddin, A. De, R. Aashish, and R. David, "Lack of online video educational resources for open colorectal surgery training," Anz Journal of Surgery, vol. 23, no. 13, pp. 523-533, 2020.

[14] T. T. Nguyen, T. T. T. Nguyen, A. V. Luong, Q. V. H. Nguyen, W. C. Liew, and B. Stantic, "Multi-label classification via labels correlation and one-dependence features on data stream," Pattern Recognition, vol. 90, 2019.

[15] D. Muthukrishna, G. Narayan, K. S. Mandel, R. Biswas, and R. Hložek, "RAPID: early classification of explosive transients using deep learning," Publications of the Astronomical Society of the Pacific, vol. 131, no. 105, pp. 118-131, 2019.

[16] H. Melyssa, H. Michael, and M. Sonya, "Educational resources for global health in otolaryngology," Otolaryngologic Clinics of North America, vol. 51, no. 3, pp. 563-574, 2018.

[17] G. M. Machado, V. Maran, G. M. Lunardi, L. K. Wives, and J. P. M. D. Oliveira, "AwARE: A framework for adaptive recommendation of educational resources," Computing, 2021.

[18] J. L. Lobo, I. Oregi, A. Bifet, and J. D. Ser, "Exploiting the stimuli encoding scheme of evolving Spiking Neural Networks for stream learning," Neural Networks, vol. 14, no. 43, pp. 124-156, 2019.

[19] H. Li, H. Xu, C. Zhou, L. Xing, and Z. Han, "Joint optimization strategy of computation offloading and resource allocation in multi-access edge computing environment," IEEE Transactions on Vehicular Technology, no. 99, 2020.

[20] R. Levy, E. Chow, B. Kwon, K. Socha, M. Mccarthy, and P. R. Turner, "SIAM education subcommittee report on undergraduate degree programs in applied mathematics," Siam Review, vol. 59, no. 1, pp. 199-204, 2017.

[21] C. Lee, S. Hong, S. Hong, and T. Kim, "Performance analysis of local exit for distributed deep neural networks over cloud and edge computing," Etri Journal, vol. 42, no. 5, 2020.

[22] O. Laureniu, D. Norbert, C. Octavian, E. Marius, and M. Costel, "Edge computing in space: field programmable gate array-based solutions for spectral and probabilistic analysis of time series," Review of Scientific Instruments, vol. 90, no. 11, p. 114501, 2019.

[23] N. Kilarkaje, H. Al-Hussaini, and R. Ettarh, "Millennial students study alone using mixed educational resources," Faseb Journal, vol. 33, no. S1, 2019.

[24] R. S. Kantar, A. R. Alfonso, E. P. Ramly, J. R. Diaz-Siso, and R. L. Flores, "Educational resources in craniofacial surgery: the case for user-friendly digital simulators," Journal of Craniofacial Surgery, vol. 31, 2020.

[25] J. H. Huh, "Reefer container monitoring system using PLCbased communication technology for maritime edge computing," Journal of Supercomputing, vol. 76, 2020.

[26] Y. Huang, X. Song, F. Ye, Y. Yang, and X. Li, "Fair and efficient caching algorithms and strategies for peer data sharing 
in pervasive edge computing environments," IEEE Transactions on Mobile Computing, p. 1, 2019.

[27] M.-P. Hosseini, T. X. Tran, D. Pompili, K. Elisevich, and H. Soltanian-Zadeh, "Multimodal data analysis of epileptic EEG and rs-fMRI via deep learning and edge computing," Artificial Intelligence in Medicine, vol. 104, Article ID 101813, 2020.

[28] X. Hong, L. Liang, X. Jie, and A. Nallanathan, "Joint task assignment and resource allocation for $\mathrm{d} 2 \mathrm{~d}$-enabled mobileedge computing," IEEE Transactions on Communications, vol. 99, 2019.

[29] S. H. Hallett and S. P. Caird, "Soil-Net: development and impact of innovative, open, online soil science educational resources," Soil Science, vol. 182, no. 5, p. 1, 2017.

[30] S. H. Hallett, S. P. Stephen, and S. P. Caird, "Soil-net," Soil Science, vol. 182, no. 5, pp. 188-201, 2017.

[31] H. M. Gomes, A. Bifet, J. Read et al., "Correction to: adaptive random forests for evolving data stream classification," $M a$ chine Learning, vol. 108, no. 10, pp. 1877-1878, 2019.

[32] M. Gairing and R. Savani, "Computing stable outcomes in symmetric additively separable hedonic games," Mathematics of Operations Research, vol. 44, 2019.

[33] R. Du, Y. Liu, L. Liu, and W. Du, "A lightweight heterogeneous network clustering algorithm based on edge computing for 5G," Wireless Networks, vol. 26, no. 3, pp. 1631-1641, 2020.

[34] S. U. Din and J. Shao, "Exploiting evolving micro-clusters for data stream classification with emerging class detection," Information Sciences, vol. 507, 2019.

[35] V. Celentano, G. Pellino, and M. G. Coleman, "Lack of online video educational resources for open colorectal surgery training," Anz Journal of Surgery, 2019.

[36] V. Camel, M. N. Maillard, N. Descharles, E. L. Roux, and I. Billault, "Open digital educational resources for selftraining chemistry lab safety rules," Journal of Chemical Education, vol. 98, no. 1, pp. 208-217, 2020.

[37] S. Ren, W. Zhu, B. Liao et al., "Selection-based resampling ensemble algorithm for nonstationary imbalanced stream data learning," Knowledge-Based Systems, vol. 163, pp. 705722, 2018.

[38] A. H. Kleynhans, D. J. Oosthuizen, and V. Hoving, "Emergency medicine educational resource use in Cape Town: modern or traditional?” Postgraduate Medical Journal, 2017.

[39] A. Boukerche and V. Soto, "Computation offloading and retrieval for vehicular edge computing," Acm Computing Surveys, vol. 53, no. 4, 2020.

[40] S. M. Alvarado and H. Feng, "Representation of dark skin images of common dermatologic conditions in educational resources: a cross-sectional analysis," Journal of the American Academy of Dermatology, vol. S0190-9622, no. 20, 31138 pages, 2020 .

[41] C. Alberto and K. Bartosz, "Evolving rule-based classifiers with genetic programming on GPUs for drifting data streams," Pattern Recognition, vol. 87, pp. 248-268, 2019.

[42] A. A. Ali, G. Adler, and M. Rapoport, "Session 303 - driving and dementia-an introduction, educational resources, and international perspectives," American Journal of Geriatric Psychiatry, vol. 25, pp. S19-S20, 2017.

[43] X. Xiong, K. Zheng, L. Lei, and L. Hou, "Resource allocation based on deep reinforcement learning in IoT edge computing," IEEE Journal on Selected Areas in Communications, no. 99, p. 1, 2020.

[44] A. Beaufort, F. Moatar, E. Sauquet, P. Loicq, and D. M. Hannah, "Influence of landscape and hydrological factors on stream-air temperature relationships at regional scale," Hydrological Processes, vol. 34, 2020. 